\title{
Comment on paper "Multi-strategy ensemble evolutionary algorithm for dynamic multi-objective optimization" by Wang and Li
}

\author{
Jasper A. Vrugt • Bruce A. Robinson • \\ James M. Hyman
}

Received: 13 April 2010 / Accepted: 15 April 2010 / Published online: 4 May 2010

(C) The Author(s) 2010. This article is published with open access at Springerlink.com

\begin{abstract}
This comment addresses the work of Wang and Li (DOI: 10.1007/s12293-009-0012-0, hereafter referred to as WL) published online on September 01, 2009 in a (special) issue of Memetic Computing. We respectfully beg to differ in opinion with WL and argue that the concept of multi-strategy evolutionary search used by WL is not particularly novel. Similar ideas have been presented elsewhere, and these publications date back to at least early 2007. For instance, in a series of papers published in PNAS (2007), SSSAJ (2008), WRR (2008), IEEE-TEVC (2009), JH (2010), Vrugt and coworkers have introduced AMALGAM, a multi-method (or ensemble) search approach to solve emerging single and multiple objective search and optimization problems. In the past few years, MATLAB, C++, Python, and R implementations of AMALGAM have been distributed extensively among researchers and practitioners in various fields of study.
\end{abstract}

Keywords Multi-method optimization - Ensemble optimizer · AMALGAM

With great interest we recently read the paper "Multi-strategy ensemble evolutionary algorithm for dynamic multiobjective optimization" by Yu Wang and Bin Li in Volume 2 Number 1, March 2010 published in the Thematic Issue on "Memetic Algorithms for Evolutionary Multi-Objective Optimization" of Memetic Computing journal [3]. This work by Wang and $\mathrm{Li}$, hereafter referred to as WL, introduces a multi-strategy ensemble multi-objective evolutionary algorithm, called MS-MOEA, to efficiently solve dynamic multiple objective optimization problems. Whereas most evolutionary approaches in the literature typically use a single algorithm for population evolution, WL propose to use

J. A. Vrugt ( $\varangle)$ · B. A. Robinson · J. M. Hyman

University of California, Irvine, CA, USA

e-mail: jasper@uci.edu genetic and differential operators jointly to create offspring and evolve a population of individuals to a limiting distribution. Indeed, results demonstrate that convergence to the Pareto solution set can be accelerated when using multiple offspring creating mechanisms simultaneously. This work extends previous work by $\mathrm{Du}$ and $\mathrm{Li}$ (DL) on single objective optimization entitled "Multi-strategy ensemble particle swarm optimization" and published in Information Sciences (2008) [4].

We would like to congratulate the authors with their work. Indeed, we believe that adaptive algorithms that do not rely on a single biological model for natural selection and adaptation, but use multiple search strategies simultaneously are the way to go to solve complex (high-dimensional) optimization problems. Multi-method algorithms (called multi-strategy by WL and DL) have the desirable ability to be able to select the most efficient search methodology for population evolution depending on the functional characteristics and properties of the response surface. A multi-method approach also provides a practical response to the No Free Lunch Theorem of [2].

The actual reason for this writing however, is not to congratulate the authors with their work, but to communicate that the ideas of WL and DL are not particularly novel, and have been published elsewhere at least a few years ago. For instance, a paper by Vrugt and Robinson (VR) "Improved evolutionary optimization from genetically adaptive multimethod search" published in the Proceedings of the National Academy of Sciences of the United States of America (2007) [1] discusses similar ideas. VR show that significant improvements to the efficiency of evolutionary search can be achieved by running multiple optimization algorithms simultaneously using new concepts of global information sharing and genetically adaptive offspring creation. They call this approach a multialgorithm, genetically adaptive 
multiobjective, or AMALGAM, method, to evoke the image of a procedure that merges the strengths of different optimization

algorithms.

We believe that it would have been appropriate for WL to discuss our ensemble optimizer or AMALGAM approach in their paper. There was plenty of opportunity to highlight and summarize our work. This would have been a rigorous scientific conduct, not only because WL acknowledged with courage in an separate e-mail discussion that they were aware of the existence of AMALGAM, but also because our PNAS paper was published at least 18 months prior to submission of their work. Obviously, one can emphasize that MS-MOEA and AMALGAM are different, yet their underlying principles remain quite similar. This should have been appropriately reported and discussed in the paper of WL.

Open Access This article is distributed under the terms of the Creative Commons Attribution Noncommercial License which permits any noncommercial use, distribution, and reproduction in any medium, provided the original author(s) and source are credited.

\section{References}

1. Vrugt JA, Robinson BA (2007) Improved evolutionary optimization from genetically adaptive multimethod search. Proc Natl Acad Sci USA 104:708-711. doi:10.1073/pnas.0610471104

2. Wolpert DH, Macready WG (1997) No free lunch theorems for optimization. IEEE Trans Evol Comput 1(1):67-82

3. Wang Y, Li B (2010) Multi-strategy ensemble evolutionary optimization for dynamic multi-objective optimization. Memetic Comput $2: 3-24$

4. Du WL, Li B (2008) Multi-strategy ensemble particle swarm optimization for dynamic optimization. Inf Sci 178(15):3096-3109
Guest Editor's Note on Technical Commentary:

Ke Tang: In my view, the comments are in general fair and reasonable. However, I would like to provide a more objective view of the whole issue. I have looked through the two papers (i.e., the PNAS and TEVC papers) in details. To be honest, the idea of combining multiple algorithms is not new at all, and probably can be dated back to 1980s. Listed below are some recent references in my mind. The literature not only covers traditional algorithms, but also EAs.

A. S. Fukunaga: "Genetic algorithm portfolios," in Proc. IEEE Congr. Evol. Comput. (CEC), La Jolla, CA, 2000, pp. 16-19.

B. A. Huberman, R. M. Lukose, and T. Hogg, "An economics approach to hard computational problems," Science, vol. 275, no. 5296, pp. 51-54, Jan. 1997.

C. P. Gomes and B. Selmon, "Algorithm portfolios," Artif. Intell., vol. 126, nos. 1-2, pp. 43-62, Feb. 2001.

The authors of the commentary have not referred to the above references in either the PNAS or TEVC paper, but that does not mean there is any indication of misconduct. Combining multiple algorithms is such a general idea that few people can claim it as their ORIGINAL contribution. In my opinion, the major point is how to make use of the general idea. If we go down to the algorithmic details, the two pieces of work are substantially different. Hence, it is understandable that $\mathrm{Yu}$ Wang and Bin $\mathrm{Li}$ did not notice it and view it as a closely related work, although referring to the PNAS paper would definitely provide readers a more comprehensive plot of the related area. 